\title{
Leptomeningeal metastasis from central nervous system tumors: A study of classification and stage in the spinal canal of 58 patients
}

\author{
ZHANG MingShan ${ }^{1}$, OU YunWei ${ }^{1}$, ZHANG HongWei ${ }^{1}$, ZHANG JunPing ${ }^{2}$, XIA Lei ${ }^{1}$, \\ QU YanMing ${ }^{1}$, WANG HaoRan ${ }^{1}$, ZHAN QiMin $^{3 *}$, SONG YongMei ${ }^{3 *} \&$ YU ChunJiang ${ }^{1 *}$ \\ ${ }^{1}$ Department of Neurosurgery, Beijing Sanbo Brain Hospital, Capital Medical University, Beijing 100093, China; \\ ${ }^{2}$ Department of Neuro-oncology, Beijing Sanbo Brain Hospital, Capital Medical University, Beijing 100093, China; \\ ${ }^{3}$ State Key Laboratory of Molecular Oncology, Cancer Institute, Cancer Hospital, Peking Union Medical College, Chinese Academy of Medical \\ Sciences, Beijing 100021, China
}

Received February 20, 2012; accepted April 9, 2012; published online June 1, 2012

\begin{abstract}
Leptomeningeal metastasis (LM) is caused by the spread of malignant tumor cells into the subarachnoid space. However, classification and staging of LM in the spinal canal is rare in the literature. The authors reviewed the records of 58 Chinese patients with LM for clinical features, neuroimaging, and treatments. Gadolinium-enhanced magnetic resonance imaging (MRI) of brain and spinal cord were performed in all patients. Removal of intracranial tumors was performed in all patients and diagnoses were confirmed by histology. The study group consisted of 58 patients, with 29 cases presenting with intraspinal symptoms. Of the 58 , 8 patients underwent intraspinal tumor removal, 8 received radiotherapy alone, 9 received chemotherapy alone, and 34 patients received combined radiochemotherapy. We classified LM into 3 types: type L or leptomeninges LM, is subdivided into 2 subtypes (subtype LI and LII (a,b)), type N or nerve root LM is subdivided into 2 subtypes (subtype NI and NII (a,b)), and type M or mixed-type LM. We also divided LM into stages of I-IV according to the symptoms and the volume of the tumor based on spinal axial MRI. Type LI LM often occurs in patients with intracranial and intraspinal tumors found simultaneously. Patients who receive surgery for intracranial tumors may present with type N LM. Surgery is suitable for patients with NI LM and LIIb LM in stages III-IV, presenting with severe spinal symptoms. The prognosis is better for type N LM than type L LM.
\end{abstract}

leptomeningeal metastasis, spinal canal, classification, stage, central nervous system tumor

Citation: Zhang M S, Ou Y W, Zhang H W, et al. Leptomeningeal metastasis from central nervous system tumors: A study of classification and stage in the spinal canal of 58 patients. Chin Sci Bull, 2012, 57: 2914-2919, doi: 10.1007/s11434-012-5262-4

Leptomeningeal metastasis (LM) is caused by the spread of malignant tumor cells into the subarachnoid space. LM was observed in 5\%-10\% of patients with solid tumors, 5\%$15 \%$ of patients with hematologic malignancies, and less than $2 \%$ of patients with primary brain tumors [1-3]. Some primary brain tumors, such as medulloblastoma and primary central nervous system lymphoma (PCNSL), have an incidence of LM as high as $40 \%$ [4]. Previous classifications and staging for the neuraxis metastasis, such as the system used by Kelly et al. [5], classified the neuraxis dissemina-

*Corresponding authors (email: zhanqimin@pumc.edu.cn; ymsong.2008@gmail.com; yuchunjiang1955@sina.com) tion of glioma into 4 types according to physiopathologic mechanisms and MRI features. Schiff and O'Neill [6] graded intramedullary metastasis into grades of $0-$ III according to the patient's ability to ambulate at the time of diagnosis. However, literatures regarding classification or staging of LM in the spinal canal are rare.

\section{Materials and methods}

Clinical findings from 58 Chinese LM patients were studied retrospectively (Table $\mathrm{S} 1$ ), including spinal symptoms and signs. Whole brain and spinal cord MRI scans were performed in all cases. Removal of intracranial tumors was 
performed in all patients and diagnoses were confirmed by histology. Of the 58 cases, there were 23 medulloblastomas, 3 primitive neuroectodermal tumors (PNET), 6 germinomas, 2 mixed germ cell tumors, 1 immature teratoma, 4 glioblastomas, 3 astrocytomas, 2 ependymomas, 3 anaplastic ependymomas, 4 pineal parenchymal tumors of intermediate differentiation (PPTID), 1 pinealoblastoma (PB), 2 esthesioneuroblastomas, 1 anaplastic meningioma, 1 pituitary adenoma, 1 choroid plexus papilloma (CPP) and 1 PCNSL.

According to growth patterns of the tumors using MRI, we classified LM into 3 types: type L or leptomeninges type, type $\mathrm{N}$ or nerve root type, and type $\mathrm{M}$ or mixed-type LM.

Type L occurs when tumor cells grow on the surface of the leptomeninges and is subdivided into 2 subtypes: subtype LI and subtype LII. LI LM occurs when tumor cells disseminate on the leptomeninges in a miliary or linear pattern without masses in the subarachnoid space. LII LM occurs when tumor cells grow in the procumbent mode. LII LM is further divided into 2 subtypes, LIIa and LIIb according to the different growth patterns. LIIa is defined as metastatic foci with an extensive distribution, while LIIb is considered to have a limited distribution (Figure 1).

Type $\mathrm{N}$ is considered to be the most metastases with more than one lesion developing in the spinal nerves, most frequently in the cauda equina. Type $\mathrm{N}$ is further divided into 2 subtypes, NI and NII. NI LM tumors grow in a nodular mode, and NII tumors are large volume tumors with a diameter greater than the height of 2 vertebral bodies. There are also two NII growth patterns. In NIIa tumors, the metastatic foci have irregular borders, and with NIIb the borders are distinct (Figure 2).

Type M, or mixed-type LM, we define as occurring when LII LM and N LM exist simultaneously (Figure 3).

We divided LM into 4 stages according to the symptoms and the volume of the tumor based on the spinal axial MRI. Stage I, also named as miliary LM, occurs when there are

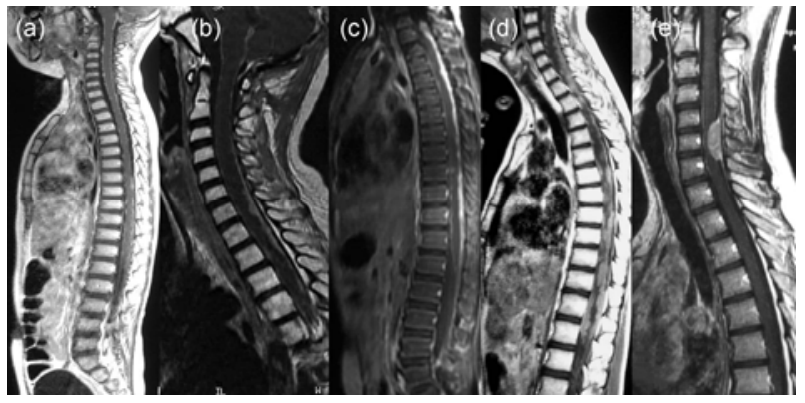

Figure 1 MRI of type LM L. (a) LI LM (case 22) MRI shows leptomeningeal linear enhancement with diffuse miliary metastases; (b) LIIa LM (case 24) MRI shows cervical and thoracic leptomeningeal thickening and enhancement with extensive distribution; (c) LIIa LM (case 26) MRI shows complete leptomeningeal thickening and enhancement; (d) LIIa LM (case 36) MRI shows complete leptomeningeal thickening and enhancement with metastatic foci filling almost the entire spinal canal; (e) LIIb LM (case 15) MRI shows a mass with a narrow base in the subarachnoid space at the level of C5-7. The growth pattern of the tumor is similar to spinal meningioma.

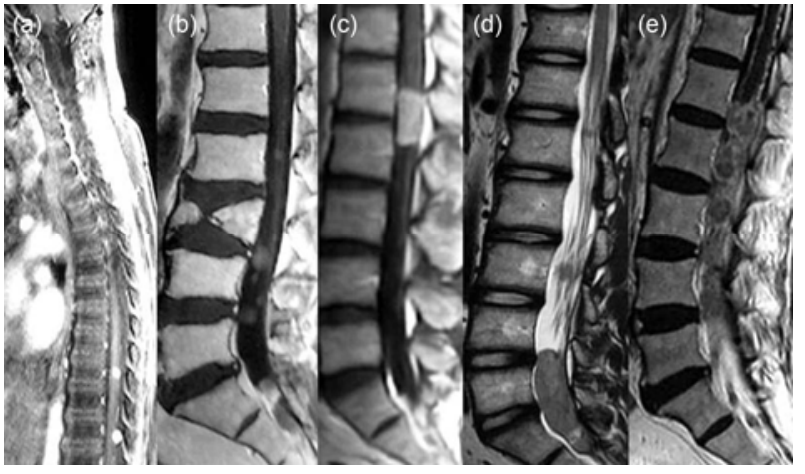

Figure 2 MRI of type LM N. (a) NI LM (case 27) showing multiple round, nodular metastases in the cauda equine; (b) NI LM (case 28) showing multiple round, nodular metastases in the cauda equine; (c) NI LM (case 54) showing a cylindrical metastasis in the cauda equine; (d) NIIb LM (case 30) showing the tumor filling the entire sacral canal with distinct borders; (e) NIIa LM (case 56) showing a giant metastasis filling the entire lumbar and sacral canal with irregular borders.

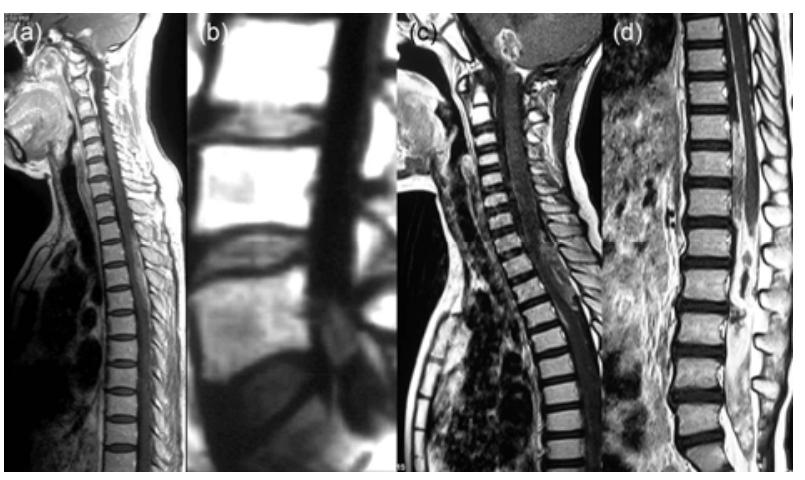

Figure 3 MRI of type M or mixed-type. (a) MRI for case 44 showing the thoracic segment containing a wide-based, procumbent LM (LIIa) growing in a creeping fashion; (b) round, nodular metastasis in the cauda equine; (c) MRI for case 46 showing that the T1-4 segments contain a limited-base, procumbent LM (LIIa); (d) T10-12 segments contain a procumbent LM (LIIa), and a giant metastasis in the cauda equina.

no intraspinal symptoms or impact on the spinal cord and spinal nerves. In stage II, the largest area of the tumors is less than $25 \%$ of the vertebral canal in the same section plane in the spinal axial MRI. In this stage, the tumor contacts or presses on the spinal cord or spinal nerves, and patients have no or mild intraspinal symptoms. In stage III, the largest area of the tumors is $25 \%-75 \%$ of the vertebral canal in the same section plane in the axial MRI. In this stage, patients suffer from intraspinal symptoms. In stage IV, the largest area of the tumors is greater than $75 \%$ of the vertebral canal in the same section plane of the axial MRI. In this stage, the spinal cord and spinal nerves are severely compressed, and serious intraspinal symptoms and paraplegia may occur (Figure 4).

\section{Results}

There were 42 males and 16 females in this group, with ages 


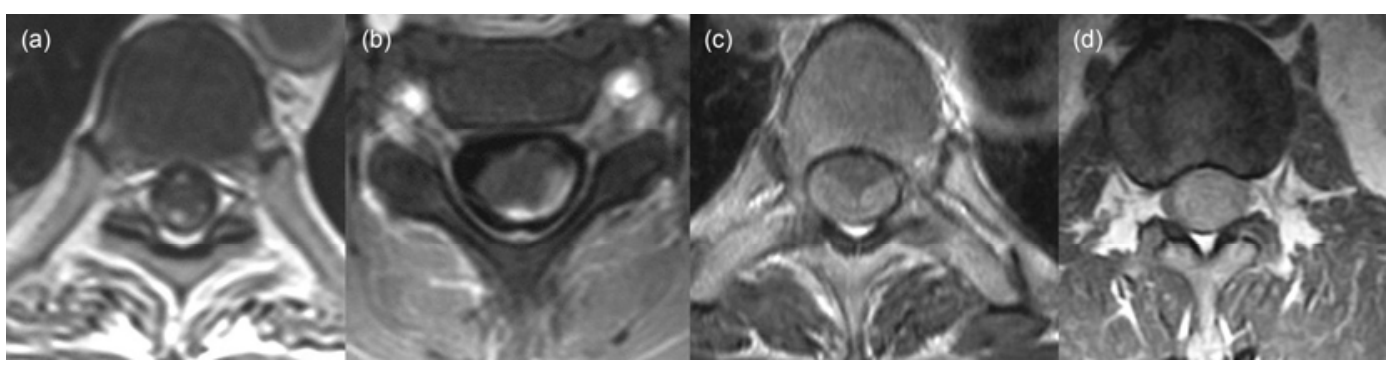

Figure 4 (a) Stage I, also called miliary LM; (b) stage II, showing the largest area of the tumors is less than $25 \%$ of the vertebral canal in the same section plane in the axial MRI; (c) stage III, showing the largest area of the tumors is less than $75 \%$ but greater than $25 \%$ of the vertebral canal in the same section plane in the axial MRI; (d) stage IV, showing the largest area of the tumors is greater than $75 \%$ of the vertebral canal in the same section plane in the axial MRI.

ranging from 2 to 71 years (average age 22.3 years). Intracranial tumors and spinal drop metastases were found simultaneously in 19 cases, spinal metastases after intracranial tumor removal were found in 39 cases from 1 to 144 months (average 32.0 months), and 29 cases presented with intraspinal symptoms.

Thirty-two cases of type L were found, and 23 cases were LI LM. In 12 cases of LI LM intracranial and intraspinal tumors were found simultaneously, accounting for $52.2 \%(12 / 23)$ of all LI cases. Patients who had previous intracranial tumor surgery were more likely to present with type $\mathrm{N}$. There were 24 cases of type $\mathrm{N}$ in our $58 \mathrm{LM}$ patients, and 21 cases of those occurred postoperatively, accounting for $87.5 \%(21 / 24)$ of type $\mathrm{N}$ (Table 1$)$.

In our patient group, 25 cases were in stage I, 5 were in stage II, 15 were in stage III and 13 were in stage IV. The symptoms and signs were different between intramedullary and subdural spinal canal lesions. Apart from 5 cases of intramedullary metastases, in the remaining 53 cases, the incidence of the intraspinal symptoms and signs was $13 \%$ $(3 / 23)$ in stage I, $25 \%(1 / 4)$ in stage II, $80 \%(12 / 15)$ in stage III and $81.8 \%(9 / 11)$ in stage IV.

Surgeries for intracranial tumor were performed in all patients and, as the adjunctive therapy, 8 patients had radiotherapy alone, 9 patients had chemotherapy alone, and 34 patients had combined radiochemotherapy. Seven patients had no any further therapy. Only 8 patients had surgery for intraspinal tumors.

All the patients were followed up postoperatively from

Table 1 LM classification in the patient group

\begin{tabular}{cllc}
\hline Type & Subtype & \multicolumn{1}{c}{ Growth features } & $\begin{array}{c}\text { Number } \\
\text { (case) }\end{array}$ \\
\hline L & LI & Miliary or/and linear & 23 \\
& LIIa & Procumbent with extensive territory & 8 \\
& LIIb & Procumbent with limited territory & 1 \\
\hline N & NI & Nodular & 19 \\
& NIIa & Giant with irregular boundary & 4 \\
& NIIb & Giant with distinct boundary & 1 \\
\hline M & LII and N & Procumbent and nodular or giant & 2 \\
\hline
\end{tabular}

0.5 to 153 months (average 26.6 months). Twenty-one patients died, 11 patients worsened, 13 patients stabilized, and 13 patients improved.

\section{Discussion and conclusions}

There are two main paths for CNS tumors to disseminate along the neuraxis. One path is direct extension arising from CNS tumors in close proximity to the leptomeninges or ependyma [4]. This occurs with medulloblastoma, ependymoma, germinoma, and pineal parenchymal tumors [7], or gliomas contiguous with the ventricular system [8]. Deciduous tumor cells travel through and then disseminate into the CSF when parenchymal tumors break through the leptomeninges or ependyma. The average interval between diagnosis of the primary brain tumor and diagnosis of CSF dissemination ranged from 5 to 14 months [9]. In two series, $33 \%-75 \%$ of patients with LM had synchronous intraparenchymal brain metastases [10,11]. This occurred in $32.8 \%$ (19/58) of the patients in our group. The volume of the metastatic foci from direct dissemination is usually very little, the foci being only slightly affected by gravity. Metastatic foci can float slowly through the CSF and grow throughout the subarachnoid space.

Another path of leptomeningeal dissemination is iatrogenic spread into the CSF during surgery for parenchymal tumors [12,13]. Mahajan et al. [14] pointed out that the incidence of LM was high if surgery was performed, and in our group the level was $67.2 \%$ (39/58). LM is particularly associated with parenchymal brain tumors located in the posterior fossa. Kitaoka et al. [15] suggested that cerebellar tumors have a higher risk than other intracranial tumors for CSF dissemination because of the proximity of the cerebellar cisterns to the subarachnoid space. In our group $62.8 \%$ (22/32) of the tumors were in the posterior fossa, and the cauda equina remained the main site of dissemination [16]. Lumbar puncture and shunting procedures may also be factors in dissemination of the tumor via the CSF pathway. Continuous CSF drainage allowed the deciduous malignant cells to accumulate in the cauda equina. A high number of tumor cells or masses could fall into the subarachnoid spac- 
es during surgery and, usually, the volume of the metastatic foci is larger than the miliary foci, and these sink into the terminal cistern because of gravity.

According to the classification of LM from CNS tumors, Zulch [17] grouped the spread of gliomas into 2 categories: multifocal and multicentric. Gururangan et al. [18] categorized pontine glioma with neuraxis spread as parenchymal (PM), subependymal (SE), or leptomeningeal (LM) based on the location of LM on MRI. Kelly et al. [5] classified the neuraxis dissemination of glioma into leptomeningeal (type I), subependymal (type II), satellite (type IIIa, IIIb), and mixed (type IV) according to physiopathological mechanisms and the MRI patterns of neuraxis dissemination. Saito et al. [19] classified the LM into a "diffuse type" of dissemination with diffuse enhancement of the subarachnoid space on spinal MRI, a "nodular type" with a nodular tumor mass, and a "mixed type" with diffuse and nodular features. Three growth patterns of LM, thin diffuse coating of the meninges, plaque-like, and nodular deposits, were described by O'Meara et al. [20]. All classifications were based on a single glioma with intracrainal metastases, and seldom contained the characteristics of LM in the spinal canal. The diffuse type is the counterpart to type $\mathrm{L}$, and the plaquelike form is a counterpart to type LII in our classification system.

All CNS tumors can involve neuraxis metastasis [21-34] (Table 2); both malignant and benign tumors. To the best of our knowledge and search of the literatures, 58 cases of LM

Table 2 Incidence of LM in different CNS tumors

\begin{tabular}{ll}
\hline \multicolumn{1}{c}{ CNS tumor } & \multicolumn{1}{c}{ Incidence of LM } \\
\hline Medulloblastoma & $27 \%-43 \%[21]$ \\
PCNSL & $42 \%[22]$ \\
Glioblastoma & $15 \%-25 \%[23]$ \\
Oligodendroglioma & $14 \%[24]$ \\
Ependymoma & $13 \%[25]$ \\
PB and PPTID & $12.9 \%[26]$ \\
Low grade astrocytoma & $3.7 \%-5.3 \%[27]$ \\
Germinoma & $0-57 \%[28]$ \\
Mixed germ cell tumor & $4 \%[29]$ \\
Meningioma & $0.1 \%[30]$ \\
Pituitary tumors & Rare 57 cases [31] \\
PRL & 15 cases \\
ACTH & 13 cases \\
GH & 9 cases \\
Nonfunctioning pituitary tumor & 17 cases \\
TSH & 1 case \\
FSH-LH & 1 case \\
Choroid plexus carcinoma & Rare 11 cases [32,33] cases [34] \\
Esthesioneuroblastoma & \\
\hline &
\end{tabular}

involving 10 types of CNS tumors is the largest group at a single institution. In our group, there were larger numbers of medulloblastoma/PNET and these involved types LI, LII, NI, and NII LM. There were a smaller number of glioblastomas/ astrocytomas, germ cell tumors, ependymomas/ana-plastic ependymomas, and pineal parenchymal tumors which involved both LI or LII, and NI or NII LM. Therefore, all types of CNS tumors have similar dissemination patterns and we included all CNS tumors together to classify the LM.

LI LM is the early stage of LM and the most common type. The cancer cells breach the leptomeninges and reach the CSF while floating along CSF pathways where they settle and grow anywhere along the neuraxis in a diffuse and multifocal manner. Progression of LI LM can follow 4 different pathways: (1) Stabilization as the LI type, with no change in the tiny metastatic foci. (2) Development from LI LM to LII LM, if the tiny metastatic foci grow and gather together to become plaque-like metastatic foci. The entire leptomeninges are gradually colonized by the tumor cells and LII LM grows in a creeping mode that is similar to the growth pattern of moss (procumbent). (3) Progression into NI LM when the tiny foci settle and grow into spinal nerves. The tiny deposits gradually grow into nodular metastatic foci. (4) Transition directly into NII LM where the deciduous tumor cells continuously fall and accumulate in the terminal cistern. Concurrently, the tumor cells grow uninterrupted and eventually the metastatic foci fill the whole lumbosacral canal. This mode of growth is similar to the formation of a coral reef. The formation process can be seen in MRIs with the developed mass seen at the bottom of the lumbosacral canal, developing mass in the middle, and the miliary tumor cells seen in the superior part of the lumbosacral canal (Figure 5(d)).

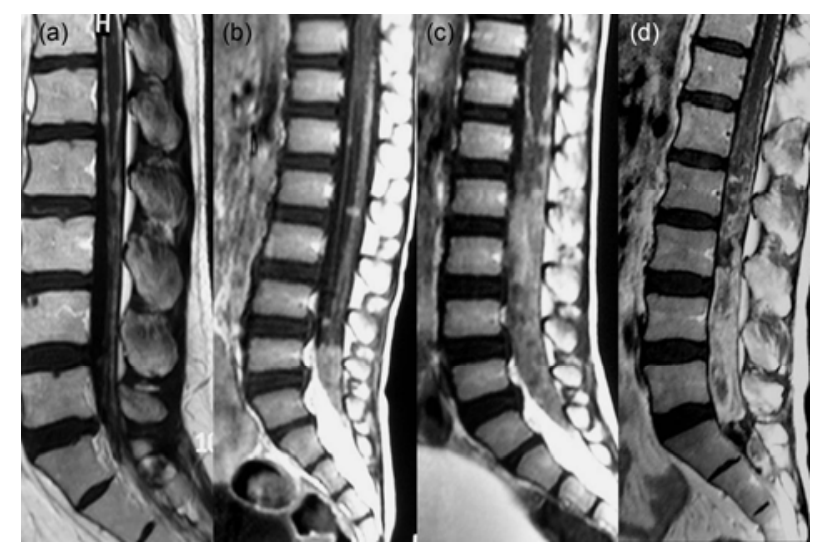

Figure 5 The process of formation of NIIa from LI LM. (a) Case 5 showing miliary metastaic foci depositing in the cauda equine; (b) and (c) case 32 MRI of the same patient at different periods of time over 1.5 months; (d) case 51 showing the miliary metastatic foci are forming into NII LM. In the MRI, the developed mass can be seen at the level of L4-S1, the developing mass can be seen at the level of L2-L3, and miliary tumor cells without a defined mass can be seen above the level of L2. 
LII LM is a rare subtype of LM described in the previous literature [35]. LII LM has two growth patterns, one that covers an extensive expanse of territory and a second that has a limited growth pattern. In the extensive growth pattern, the metastatic foci may possess multiple growth centers which, in the terminal stage, could stretch and cover the entire leptomeninges with tumor cells. With the limited growth pattern, the metastatic foci grow from a single center and the focus is usually solitary in the leptomeninges. This limited pattern is similar to spinal meningioma with a broad base and tail sign.

Type $\mathrm{N}$ of LM usually occurs following craniotomy. The tumor cells or masses fall into the subarachnoid space and the volume of the metastatic foci is usually larger than miliary foci. The metastatic foci sink into the terminal cistern due to their large volume and the effects of gravity. NI LM may remain in the same stage, or the nodular metastatic foci can grow into giant metastatic foci. NII LM can develop from LI LM or NI LM and the difference is that those originating from LI LM have irregular boundaries (NIIa), while those arising from NI have distinct boundaries (NIIb) (Figures 5(d), 6(c)).

Patients with LM should receive complete craniospinal MRI to properly determine the treatment strategy and assess the prognosis. Once the tumor involves any part of the leptomeninges, the tumor cells could begin to spread through the CSF to the whole neuraxis. Thus, leptomeningeal metastasis, although sometimes limited to few localized areas, is usually considered a diffuse disease of the CNS [36]. Patients with brain tumors are usually scanned with intracranial MRI, and complete spinal MRI is not part of the routine

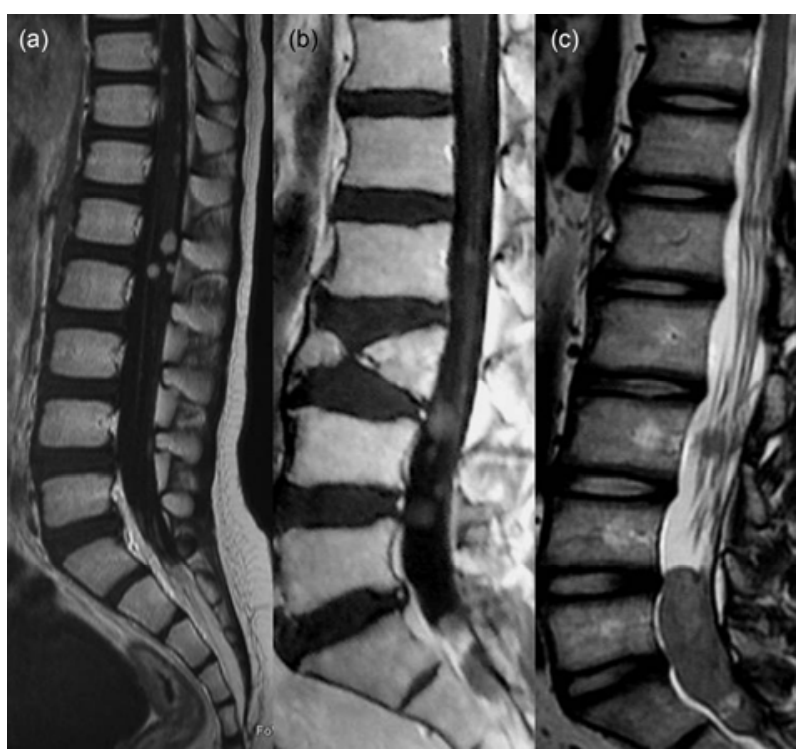

Figure 6 MRI showing the formation of NIIb from NI LM. (a) (case 40) The nodular metastatic foci are very small; (b) (case 28) large nodular metastatic foci in the cauda equine; (c) (case 30) nodular metastatic mass growing uninterrupted into a giant mass in the sacral canal. examination. However, complete spinal MRI should be carried out in the following situations: (1) Leptomeningeal focal, diffuse or nodular enhancements can be seen on the cerebral and cerebellar folia, basal cisterns, tentorium, and the ventricular ependymal surface in the cranial MRI. (2) Patients present with symptoms and signs that cannot be explained by the intracranial tumors. (3) Patients suffer from brain tumors that are prone to disseminate through the CSF, such as medulloblastoma.

Spinal surgeries are not suitable for subtypes LI LM as the metastases are tiny and multiple, and LIIa and NII LM as the metastasis is extensively distributed. Spinal surgeries are suitable for LIIb LM and NI LM because the metastases are localized. Eight cases received excision of intraspinal tumors in this group and all 8 cases were stages III-IV. The symptoms improved in 7 cases with NI, LIIb and M (NI mixed with LIIb) types. However, in 1 case of astrocytoma (case 1) LIIa subtype, spinal surgeries were performed 3 times to remove all the spinal canal metastases. In this case, the prognosis was poor for spinal injury and instability of the vertebral column. Therefore, spinal surgery should not be performed if the metastasis is dispersed in LM, unless patients with NI and LIIb LM in stages III-IV present with severe symptoms related to spinal compression. Surgical intervention cannot remove all the intraspinal tumors. Therefore, chemotherapy and radiotherapy should be the major methods for systemic treatment. All types and stages LM were suitable for chemotherapy or/and radiotherapy if the tumors types were suitable for these therapies. Spinal imaging helps to determine the placement of the inferior field edge for spinal radiation [37].

LM is the terminal stage of cancer, the prognosis for patients with LM is very poor, and untreated patients usually die within months. Median survival of treated patients is 4-5 months but varies depending on primary tumor types [38]. The cause of death in patients with LM is meningeal (neurological) tumor progression (5\%-87\%), treatmentrelated toxicity $(0-15 \%)$, or other non-neoplastic diseases $(0-29 \%)$ [39]. The prognosis is related to the primary tumor, the location of the tumor, and the treatments involved. For example, germinomas have an optimistic prognosis as they are sensitive to chemotherapy and radiotherapy. There was only 1 case of death due to germinoma in our group. The survival rate is higher in type N LM than for type L LM. The reasons are that type $\mathrm{N}$ usually occurs in the cauda equina, an area that cannot produce lethal neurological dysfunction. Furthermore, type N LM is suitable for surgery. Saito et al. [19] reported one patient with nodular type glioma who survived for 20 months after spinal dissemination. This type of dissemination may be more controllable with local intensive treatment. Type L diffuses not only into the spinal canal, but also into the entire neuraxis. In our 23 medulloblastoma patients, 11 died, and among them, only 1 was type $\mathrm{N}$; the other 10 cases were type $\mathrm{L}$. 
1 Posner J B. Neurologic Complications of Cancer. Philadelphia, PA: F.A. Davis Company, 1995

2 Groves M D. The pathogenesis of neoplastic meningitis. Curr Oncol Rep, 2003, 5: 15-23

3 Seute T, Leffers P, ten Velde G P, et al. Leptomeningeal metastases from small cell lung carcinoma. Cancer, 2005, 104: 1700-1705

4 Kesari S, Batchelor T T. Leptomeningeal metastases. Neurol Clin, 2003, 21: 25-66

5 Kelly C B, Mauricio C N, Ricardo R, et al. Patterns of neuraxis dissemination of gliomas: Suggestion of a classification based on magnetic resonance imaging findings. Surg Neurol, 2006, 65: 472-477

6 Schiff D, O'Neill B P. Intramedullary spinal cord metastases: Clinical features and treatment outcome. Neurology, 1996, 47: 906-912

7 Boyle R, Thomas M, Adams J H. Diffuse involvement of the leptomeninges by tumor-A clinical and pathological study of 63 cases. Postgrad Med J, 1980, 56: 149-158

8 Vertosick F T, Selker R G. Brain stem and spinal metastases of supratentorial glioblastoma multiforma: A clinical series. Neurosurgery, 1990, 27: 516-522

9 Natale M, Spennato P, Savarese L, et al. Anaplastic oligodendroglioma presenting with drop metastases in the cauda equina. Clin Neurol Neurosurg, 2005, 107: 417-420

10 Wasserstrom W R, Glass J P, Posner J B. Diagnosis and treatment of leptomeningeal metastases from solid tumors: Experience with 90 patients. Cancer, 1982, 49: 759-772

11 Glass J P, Melamed M, Chernik N L, et al. Malignant cells in cerebrospinal fluid (CSF): The meaning of a positive CSF cytology. Neurology, 1979, 29: 1369-1375

12 Norris L K, Grossman S A, Olivi A. Neoplastic meningitis following surgical resection of isolated cerebellar metastasis: A potentially preventable complication. J Neurooncol, 1997, 32: 215-223

13 van der Ree T C, Dippel D W, Avezaat C J, et al. Leptomeningeal metastasis after surgical resection of brain metastases. J Neurol Neurosurg Psychiatry, 1999, 66: 225-227

14 Mahajan A, Borden J, Tsai J S. Carcinomatous meningitis: Are surgery and gamma knife radiosurgery treatment risk factors? J Neurosurg, 2002, 97: 441-444

15 Kitaoka K, Abe H, Aida T, et al. Follow-up study on metastatic cerebellar tumor surgery - Characteristic problems of surgical treatment. Neurol Med Chir, 1990, 30: 591-598

16 Wippold F J, Smirniotopoulos J G, Pilgram T K. Lesions of the cauda equina: A clinical and pathology review from the Armed Forces Institute of Pathology. Clin Neurol Neurosurg, 1997, 99: 229-234

17 Zulch K J. Brain Tumors. Their Biology and Pathology. 3rd ed. Berlin: Springer, 1986

18 Gururangan S, McLaughlin C A, Brashears J, et al. Incidence and patterns of neuraxis metastases in children with diffuse pontine glioma. J Neurooncol, 2006, 77: 207-212

19 Saito R, Kumabe T, Jokura H, et al. Symptomatic spinal dissemina- tion of malignant astrocytoma. J Neurooncol, 2003, 61: 227-235

20 O'Meara W P, Borkar S A, Stambuk H E, et al. Leptomeningeal metastasis. Curr Probl Cancer, 2007, 31: 367-424

21 Eran A, Ozturk A, Aygun N, et al. Medulloblastoma: Atypical CT and MRI findings in children. Pediatr Radiol, 2010, 40: 1254-1262

22 Balmaceda C, Gaynor J J, Sun M, et al. Leptomeningeal tumor in primary central nervous system lymphoma: Recognition, significance, and implications. Ann Neurol, 1995, 38: 202-209

23 Erlich S S, Davis R L. Spinal subarachnoid metastasis from primary intracranial glioblastoma multiforme. Cancer, 1978, 42: 2854-2864

24 Enghelard H H. Current diagnosis and treatment of oligodendroglioma. Neurosurg Focus, 2002, 12: E2

25 Stüben G, Stuschke M, Kroll M, et al. Postoperative radiotherapy of spinal and intracranial ependymomas: Analysis of prognostic factors. Radiother Oncol, 1997, 45: 3-10

26 Lutterbach J, Fauchon F, Schild S E, et al. Malignant pineal parenchymal tumors in adult patients: Patterns of care and prognostic factors. Neurosurgery, 2002, 51: 44-56

27 Figueiredo E G, Matsushita H, Machado A G, et al. Leptomeningeal dissemination of pylocytic astrocytoma at diagnosis in childhood: Two cases report. Arq Neuropsiquiatr, 2003, 61: 842-847

28 Kahler R J, Coyne T. Treatment of an intracranial germinoma spinal metastasis. J Clin Neurosci, 1999, 6: 171-174

29 Schild S E, Scheithauer B W, Haddock M G, et al. Histologically confirmed pineal tumors and other germ cell tumors of the brain. Cancer, 1996, 78: 2564-2571

30 Pasquier B, Gasnier F, Pasquier D, et al. Papillary meningioma. Clinicopathologic study of seven cases and review of the literature. Cancer, 1986, 58: 299-305

31 Kaltsas G A, Grossman A B. Malignant pituitary tumors. Pituitary, 1998, 1: 69-81

32 Akil H, Coupe N J, Singh J. Spinal deposits of a benign choroid plexus papilloma. J Clin Neurosci, 2008, 15: 708-712

33 Kaptanoglu E, Tun K, Celikmez R C, et al. Spinal drop metastasis of choroid plexus papilloma. J Clin Neurosci, 2007, 14: 381-383

34 Arnold P M, Habib A, Newell K, et al. Esthesioneuroblastoma metastatic to the thoracic intradural and extradural space. Spine J, 2009, 9: e1-e5

35 Chawla A, Emmanuel J V, Seow W T, et al. Paediatric PNET: Pre-surgical MRI features. Clin Radiol, 2007, 62: 43-52

36 Stacey L B, David G P. Advances in the treatment of meningeal cancers. Crit Rev Oncol Hematol, 1995, 20: 87-98

37 Dunbar S F, Barnes P D, Tarbell N J. Radiologic determination of the caudal border of the spinal field in cranial spinal irradiation. Int J Radiat Oncol Biol Phys, 1993, 26: 669-673

38 Herrlinger U, Förschler H, Küker W, et al. Leptomeningeal metastasis: Survival and prognostic factors in 155 patients. J Neurol Sci, 2004, 223: 167-178

39 Gleissner B, Chamberlain M C. Neoplastic meningitis. Lancet Neurol, 2006, 5: 443-452

Open Access This article is distributed under the terms of the Creative Commons Attribution License which permits any use, distribution, and reproduction in any medium, provided the original author(s) and source are credited.

\section{Supporting Information}

Table S1 Clinical data from 58 cases of Leptomeningeal Metastasis (LM)

The supporting information is available online at csb.scichina.com and www.springerlink.com. The supporting materials are published as submitted, without typesetting or editing. The responsibility for scientific accuracy and content remains entirely with the authors. 